Network Working Group

Request for Comments: 1514
P. Grillo

Network Innovations

Intel Corporation

S. Waldbusser

Carnegie Mellon University

September 1993

Host Resources MIB

Status of this Memo

This RFC specifies an Internet standards track protocol for the Internet community, and requests discussion and suggestions for improvements. Please refer to the current edition of the "Internet Official Protocol standards" for the standardization state and status of this protocol. Distribution of this memo is unlimited.

Abstract

This memo defines a MIB for use with managing host systems. The term "host" is construed to mean any computer that communicates with other similar computers attached to the internet and that is directly used by one or more human beings. Although this MIB does not necessarily apply to devices whose primary function is communications services (e.g., terminal servers, routers, bridges, monitoring equipment), such relevance is not explicitly precluded. This MIB instruments attributes common to all internet hosts including, for example, both personal computers and systems that run variants of Unix.

Table of Contents

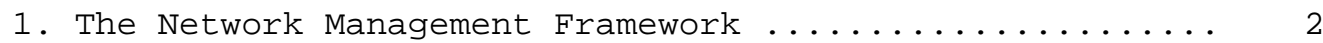

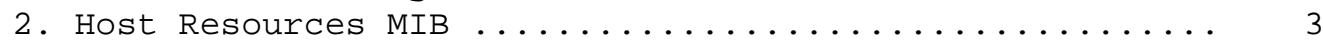

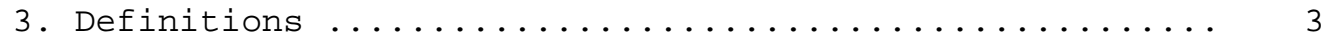

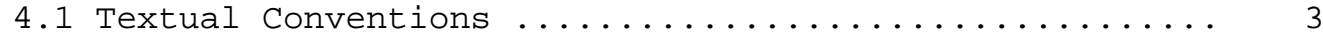

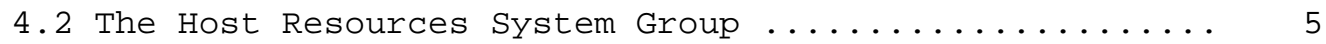

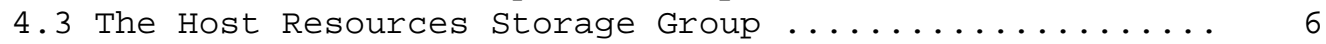

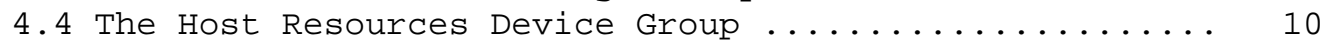

4.5 The Host Resources Running Software Group .......... 25

4.6 The Host Resources Running Software Performance

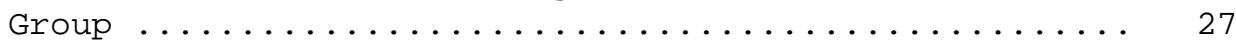

4.7 The Host Resources Installed Software Group ........ 29

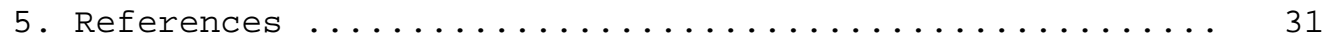

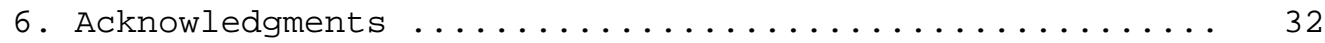

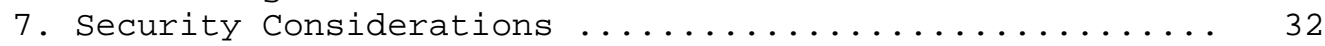

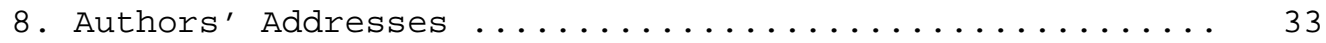

Grillo \& Waldbusser

[Page 1] 
1. The Network Management Framework

The Internet-standard Network Management Framework consists of three components. They are:

STD 16, RFC 1155 [1] which defines the SMI, the mechanisms used for describing and naming objects for the purpose of management. STD 16, RFC 1212 [2] defines a more concise description mechanism, which is wholly consistent with the SMI.

STD 17, RFC 1213 [3] which defines MIB-II, the core set of managed objects for the Internet suite of protocols.

STD 15, RFC 1157 [4] which defines the SNMP, the protocol used for network access to managed objects.

The Framework permits new objects to be defined for the purpose of experimentation and evaluation.

Managed objects are accessed via a virtual information store, termed the Management Information Base or MIB. Within a given MIB module, objects are defined using STD 16, RFC 1212's OBJECT-TYPE macro. At a minimum, each object has a name, a syntax, an access-level, and an implementation-status.

The name is an object identifier, an administratively assigned name, which specifies an object type. The object type together with an object instance serves to uniquely identify a specific instantiation of the object. For human convenience, we often use a textual string, termed the object descriptor, to also refer to the object type.

The syntax of an object type defines the abstract data structure corresponding to that object type. The ASN.1[5] language is used for this purpose. However, RFC 1155 purposely restricts the ASN.1 constructs which may be used. These restrictions are explicitly made for simplicity.

The access-level of an object type defines whether it makes "protocol sense" to read and/or write the value of an instance of the object type. (This access-level is independent of any administrative authorization policy.)

The implementation-status of an object type indicates whether the object is mandatory, optional, obsolete, or deprecated. 


\section{Host Resources MIB}

The Host Resources MIB defines a uniform set of objects useful for the management of host computers. Host computers are independent of the operating system, network services, or any software application.

The Host Resources MIB defines objects which are common across many computer system architectures.

In addition, there are objects in MIB-II [3] which also provide host management functionality. Implementation of the System and Interfaces groups is mandatory for implementors of the Host Resources MIB.

3. Definitions

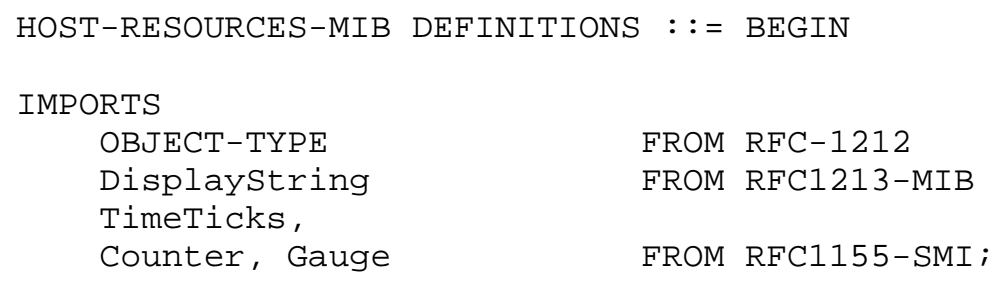


-- versions of a product should be registered under a subtree

-- distinct to that product. With this strategy, a management

-- station may uniquely determine the manufacturer and/or model of a

-- product whose productID is unknown to the management station.

-- Objects of this type may be useful for inventory purposes or for

-- automatically detecting incompatibilities or version mismatches

-- between various hardware and software components on a system.

ProductID : := OBJECT IDENTIFIER

-- unknownProduct will be used for any unknown ProductID

-- unknownProduct OBJECT IDENTIFIER : := $\left\{\begin{array}{ll}0 & 0\end{array}\right\}$

-- For example, the product ID for the ACME $486066 \mathrm{MHz}$ clock doubled

-- processor might be:

-- enterprises.acme.acmeProcessors.a4860DX2.MHz66

-- A software product might be registered as:

-- enterprises.acme.acmeOperatingsystems.acmedos.six (6). one (1)

\begin{tabular}{|c|c|c|c|c|c|}
\hline Dat & AndTime & $=\mathrm{OCTE}$ & ET STRINC & $(\operatorname{SIZE} \quad(8 \mid 11))$ & \\
\hline-- & A da & te-tin & ne specif & ication for the local time & of day. \\
\hline-- & This & data & type is & intended to provide a consis & stent \\
\hline-- & meth & lod of & reportin & 9 date information. & \\
\hline-- & & & & & \\
\hline-- & & field & octets & contents & range \\
\hline-- & & & & & \\
\hline-- & & 1 & $1-2$ & year & $0 . .65536$ \\
\hline-- & & & & (in network byte order) & \\
\hline-- & & 2 & 3 & month & $1 \ldots 12$ \\
\hline-- & & 3 & 4 & day & $1 \ldots 31$ \\
\hline-- & & 4 & 5 & hour & 0.23 \\
\hline-- & & 5 & 6 & minutes & 0.59 \\
\hline-- & & 6 & 7 & seconds & 0.60 \\
\hline-- & & & & (use 60 for leap-second) & \\
\hline-- & & 7 & 8 & deci-seconds & $0 \ldots 9$ \\
\hline-- & & 8 & 9 & direction from UTC & $"+"$ / " - " \\
\hline-- & & & & (in ascii notation) & \\
\hline-- & & 9 & 10 & hours from UTC & $0 \ldots 11$ \\
\hline-- & & 10 & 11 & minutes from UTC & 0.59 \\
\hline-- & & & & & \\
\hline-- & & Note $t$ & that if $c$ & nly local time is known, the & en \\
\hline-- & & timezo & one infor & mation (fields 8-10) is not & present. \\
\hline Int & rnationald & isplay & ystring : & $:=$ OCTET STRING & \\
\hline-- & This data & type & is used $t$ & o model textual information & in some \\
\hline-- & character & set. & A networ & $\mathrm{k}$ management station should & use a local \\
\hline-- & algorithm & to det & Eermine & hich character set is in use & e and how it \\
\hline-- & should be & displa & ayed. No & te that this character set $\mathrm{m}$ & may be encodec \\
\hline & & than & & per symbol, but will most 0 & often be NVT \\
\hline
\end{tabular}


- ASCII.

-- The Host Resources System Group

$--$

-- Implementation of this group is mandatory for all host systems. hrSystemUptime OBJECT-TYPE

SYNTAX TimeTicks

ACCESS read-only

STATUS mandatory

DESCRIPTION

"The amount of time since this host was last

initialized. Note that this is different from

sysUpTime in MIB-II [3] because sysUpTime is the

uptime of the network management portion of the system."

$::=\{$ hrSystem 1$\}$

hrsystemDate OBJECT-TYPE

SYNTAX DateAndTime

ACCESS read-write

STATUS mandatory

DESCRIPTION

"The host's notion of the local date and time of

day."

$::=\{$ hrSystem 2$\}$

hrSystemInitialLoadDevice OBJECT-TYPE

SYNTAX INTEGER (1..2147483647)

ACCESS read-write

STATUS mandatory

DESCRIPTION

"The index of the hrDeviceEntry for the device from

which this host is configured to load its initial

operating system configuration."

$::=\{$ hrsystem 3$\}$

hrSystemInitialLoadParameters OBJECT-TYPE

SYNTAX InternationalDisplaystring (SIZE (0..128))

ACCESS read-write

STATUS mandatory

DESCRIPTION

"This object contains the parameters (e.g. a

pathname and parameter) supplied to the load device

when requesting the initial operating system

configuration from that device."

$::=\{$ hrsystem 4$\}$ 


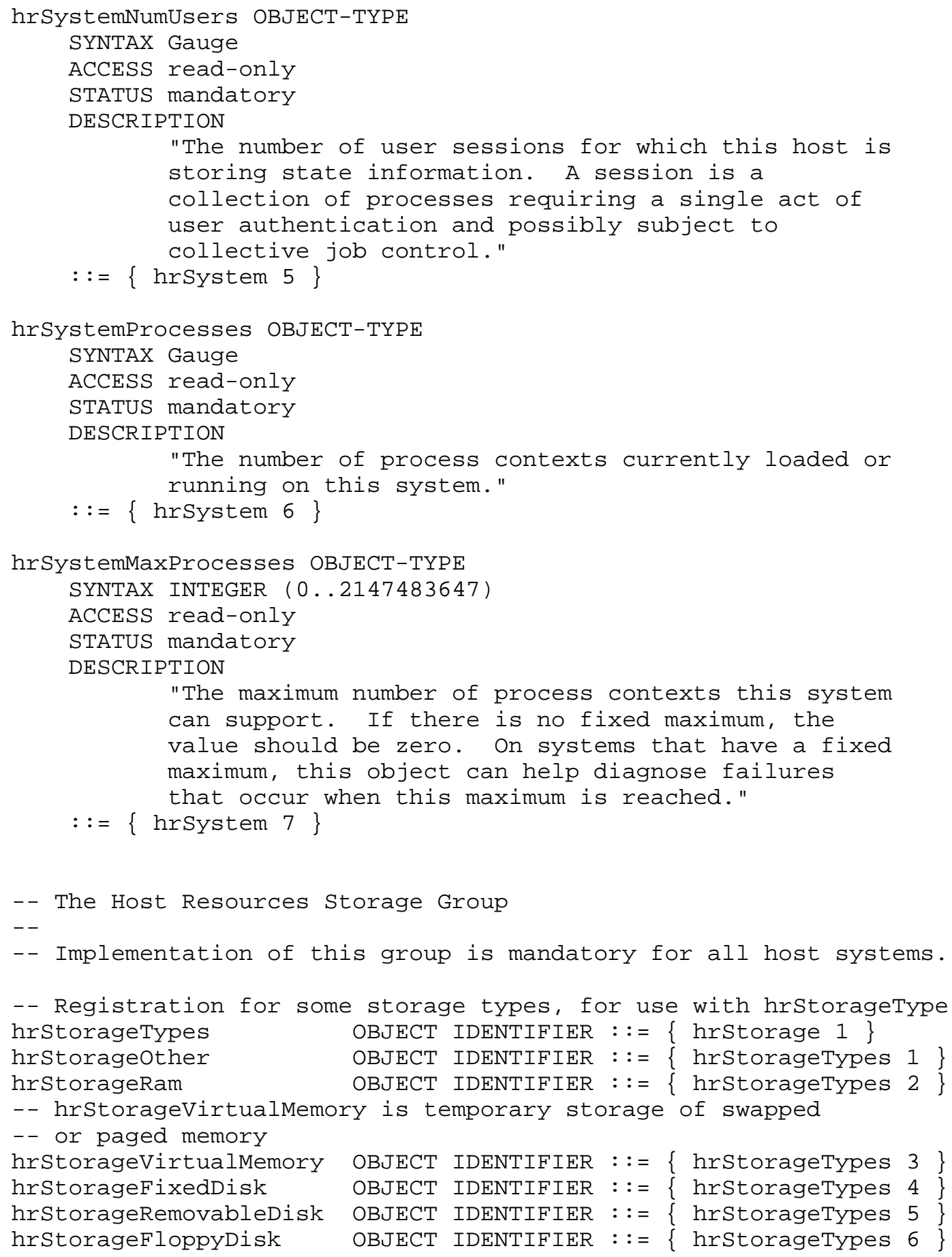




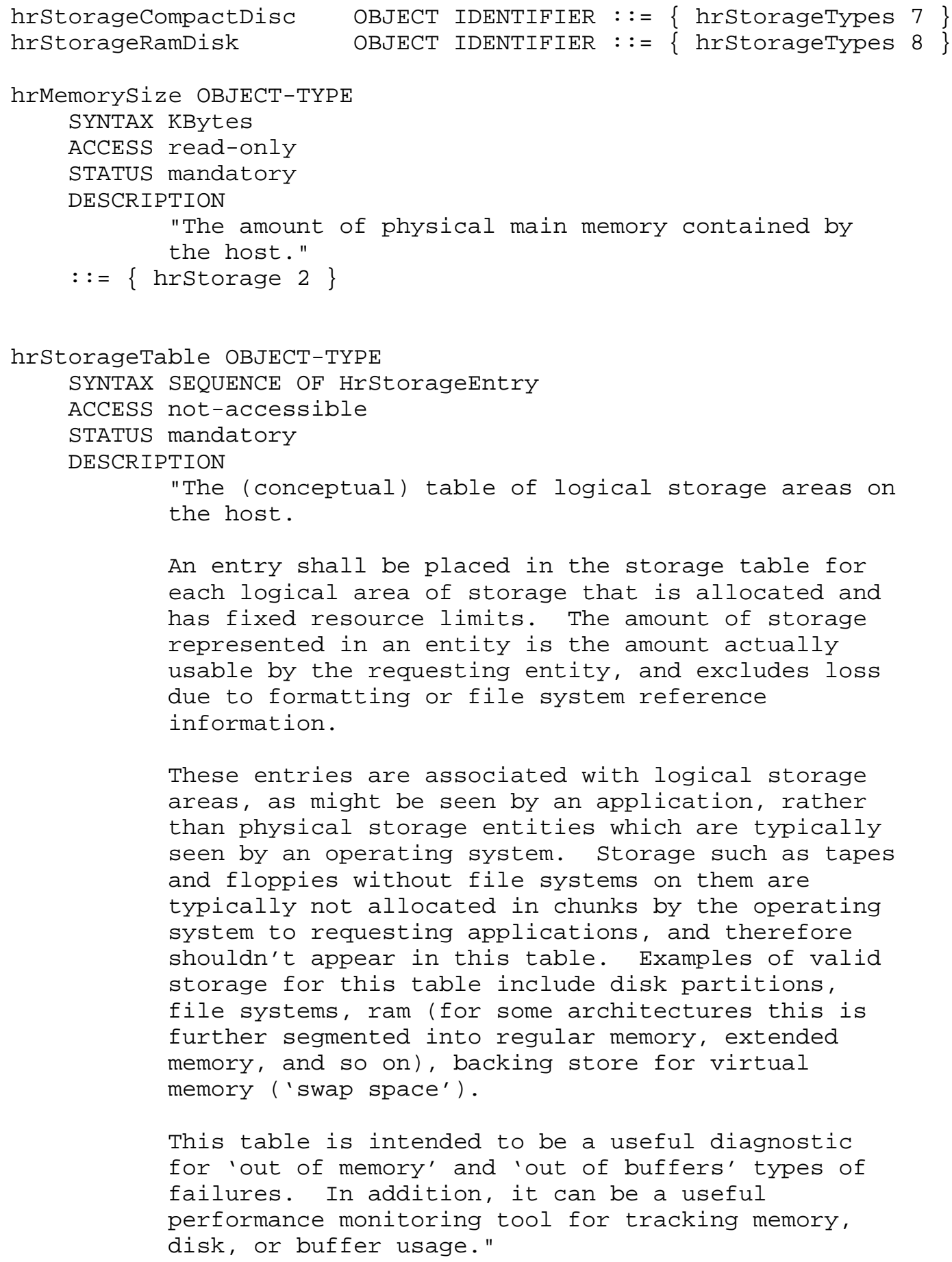




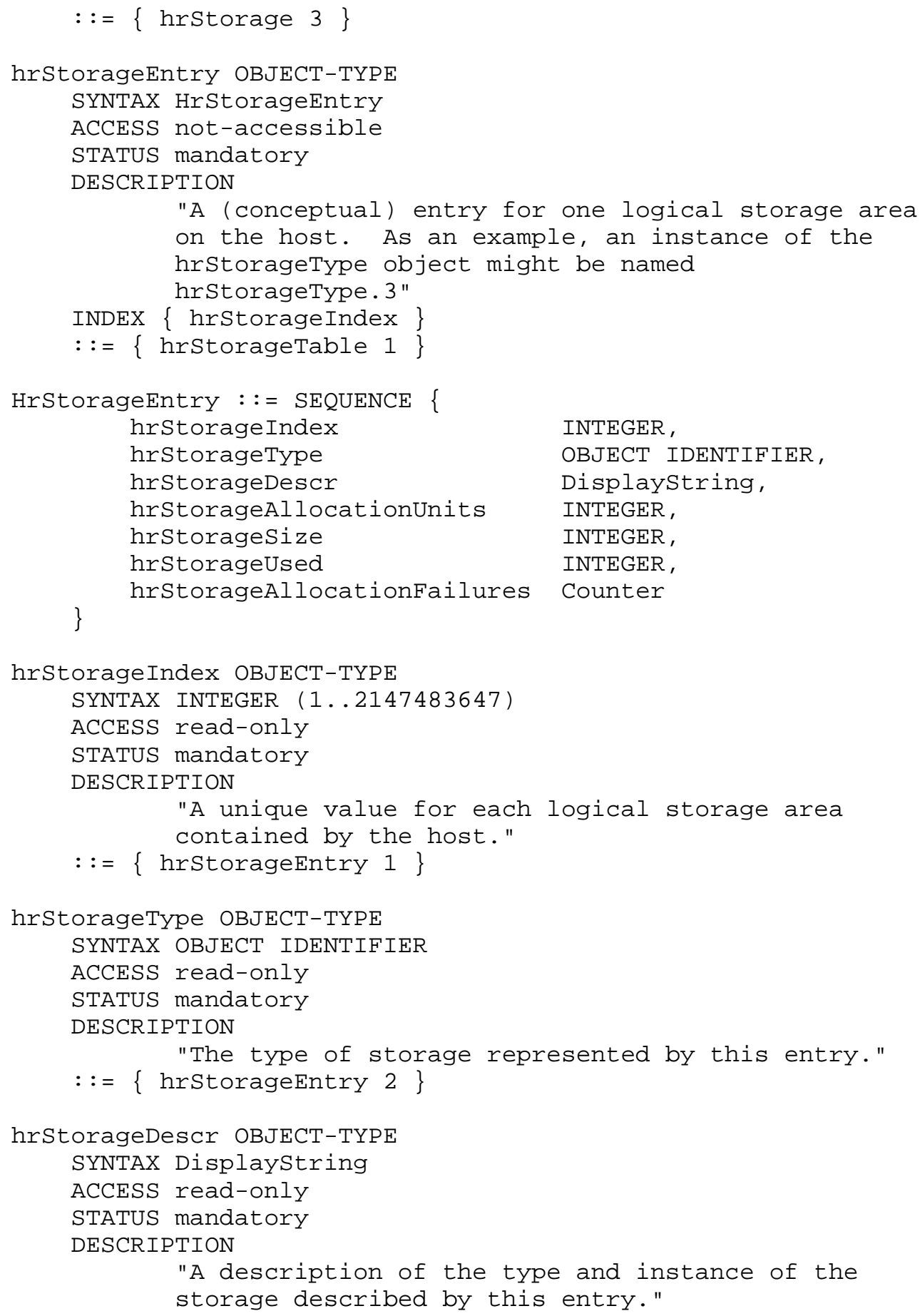


$::=\{$ hrStorageEntry 3$\}$

hrStorageAllocationUnits OBJECT-TYPE

SYNTAX INTEGER (1..2147483647)

ACCESS read-only

STATUS mandatory

DESCRIPTION

"The size, in bytes, of the data objects allocated

from this pool. If this entry is monitoring

sectors, blocks, buffers, or packets, for example, this number will commonly be greater than one. Otherwise this number will typically be one."

$::=\{$ hrStorageEntry 4$\}$

hrStorageSize OBJECT-TYPE

SYNTAX INTEGER (0..2147483647)

ACCESS read-write

STATUS mandatory

DESCRIPTION

"The size of the storage represented by this entry, in units of hrStorageAllocationUnits."

$::=\{$ hrStorageEntry 5$\}$

hrStorageUsed OBJECT-TYPE

SYNTAX INTEGER (0..2147483647)

ACCESS read-only

STATUS mandatory

DESCRIPTION

"The amount of the storage represented by this

entry that is allocated, in units of

hrStorageAllocationUnits."

$::=\{$ hrStorageEntry 6$\}$

hrStorageAllocationfailures OBJECT-TYPE

SYNTAX Counter

ACCESS read-only

STATUS mandatory

DESCRIPTION

"The number of requests for storage represented by this entry that could not be honored due to not enough storage. It should be noted that as this object has a SYNTAX of Counter, that it does not have a defined initial value. However, it is recommended that this object be initialized to zero."

$::=\{$ hrStorageEntry 7$\}$ 


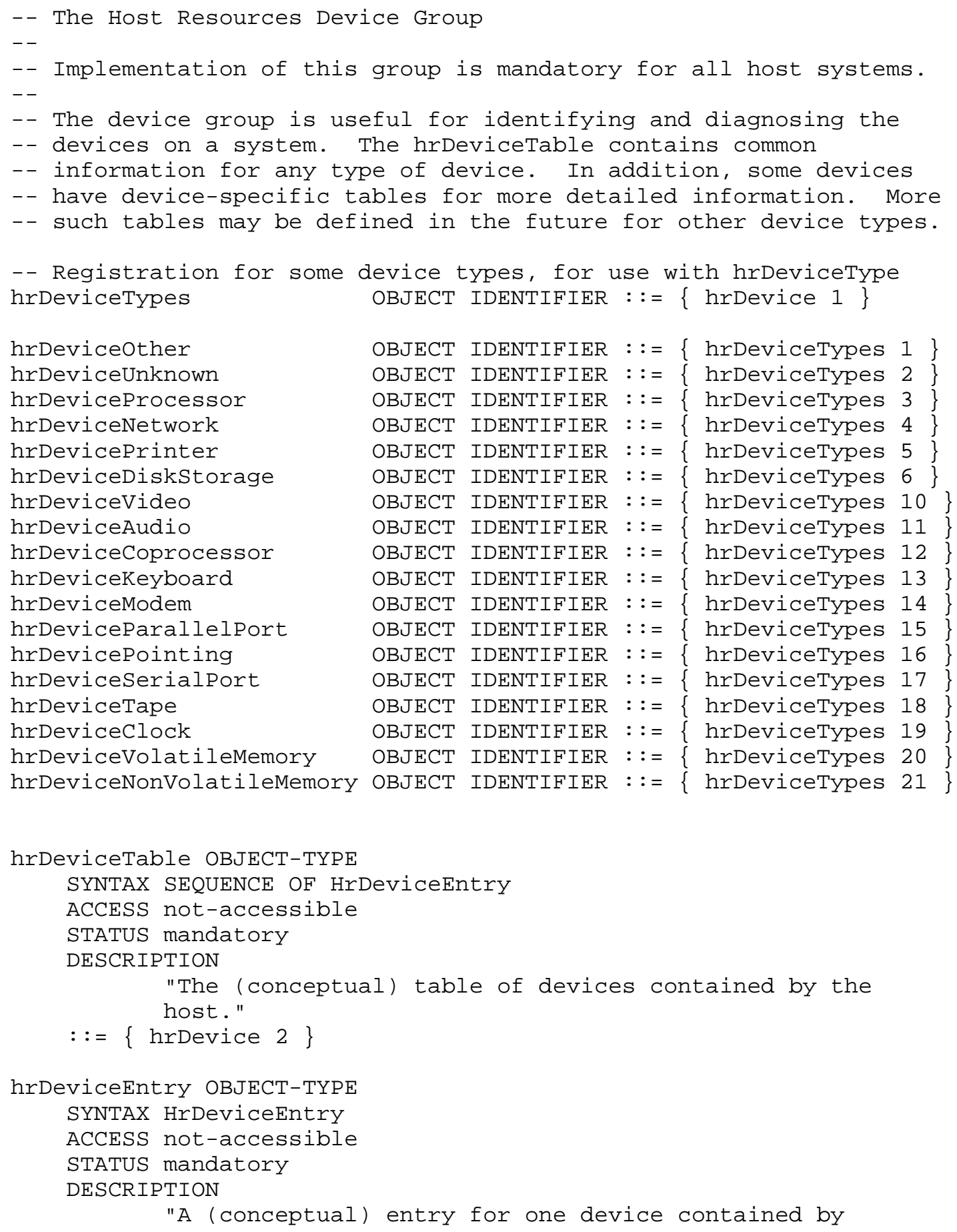




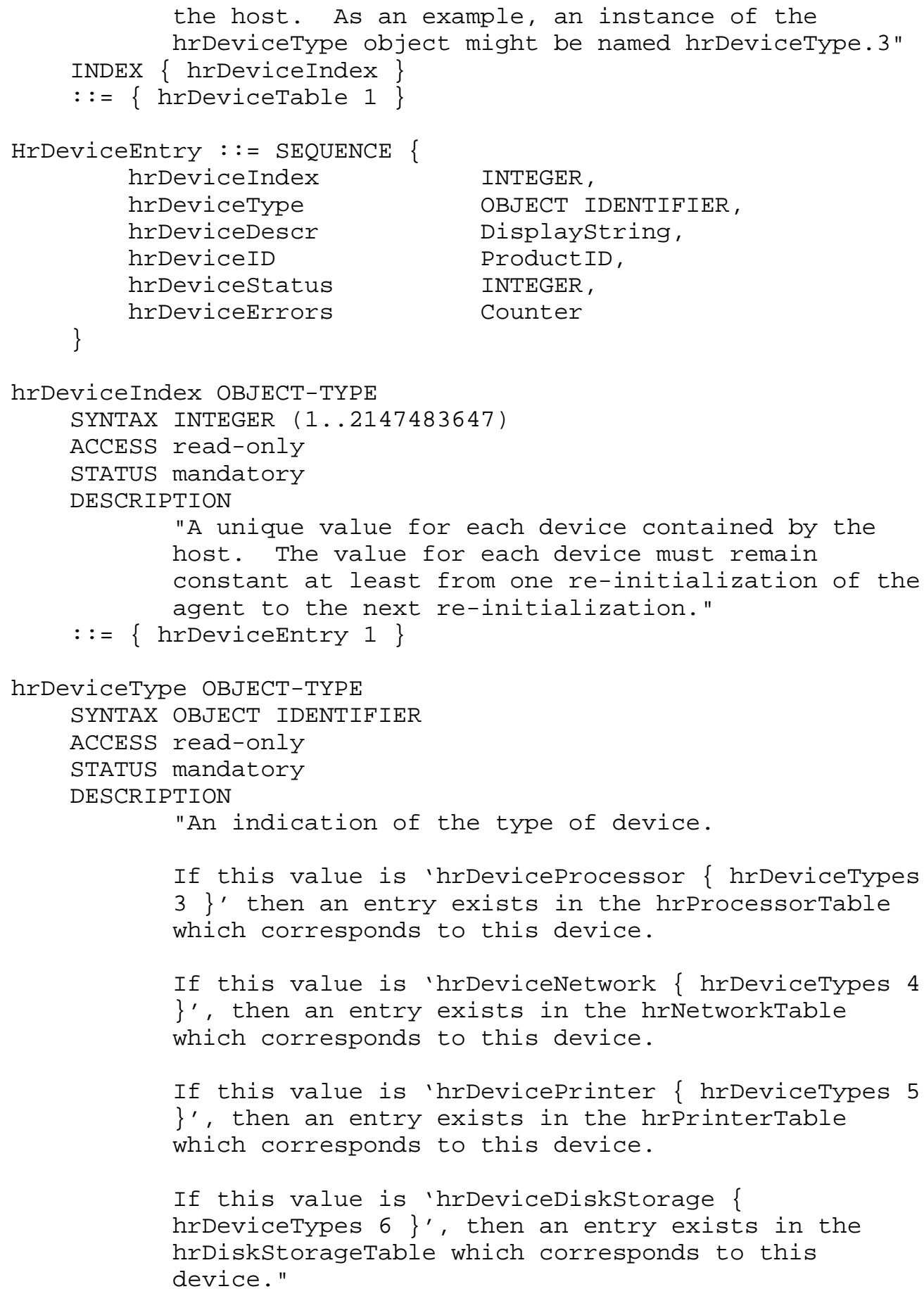




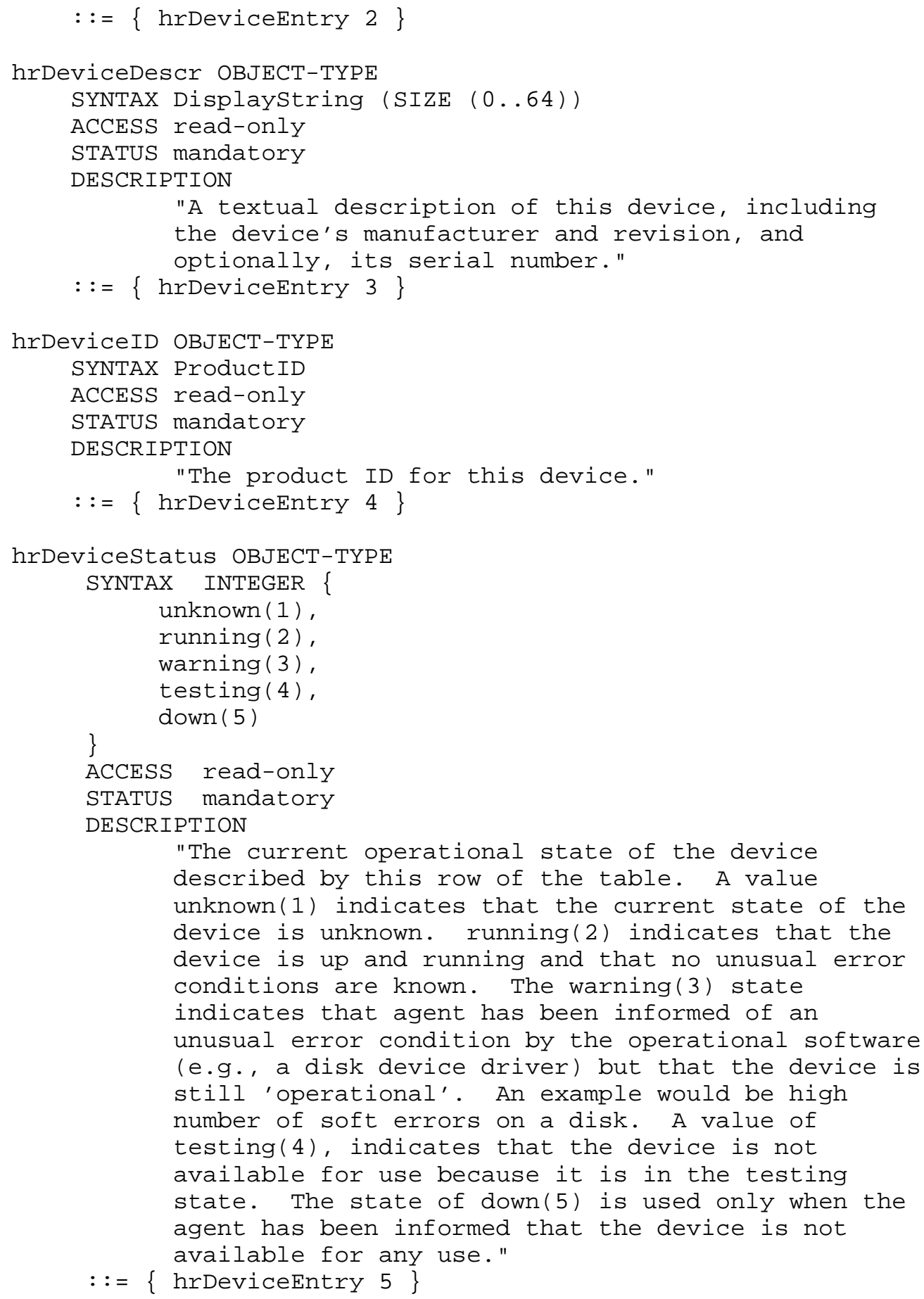




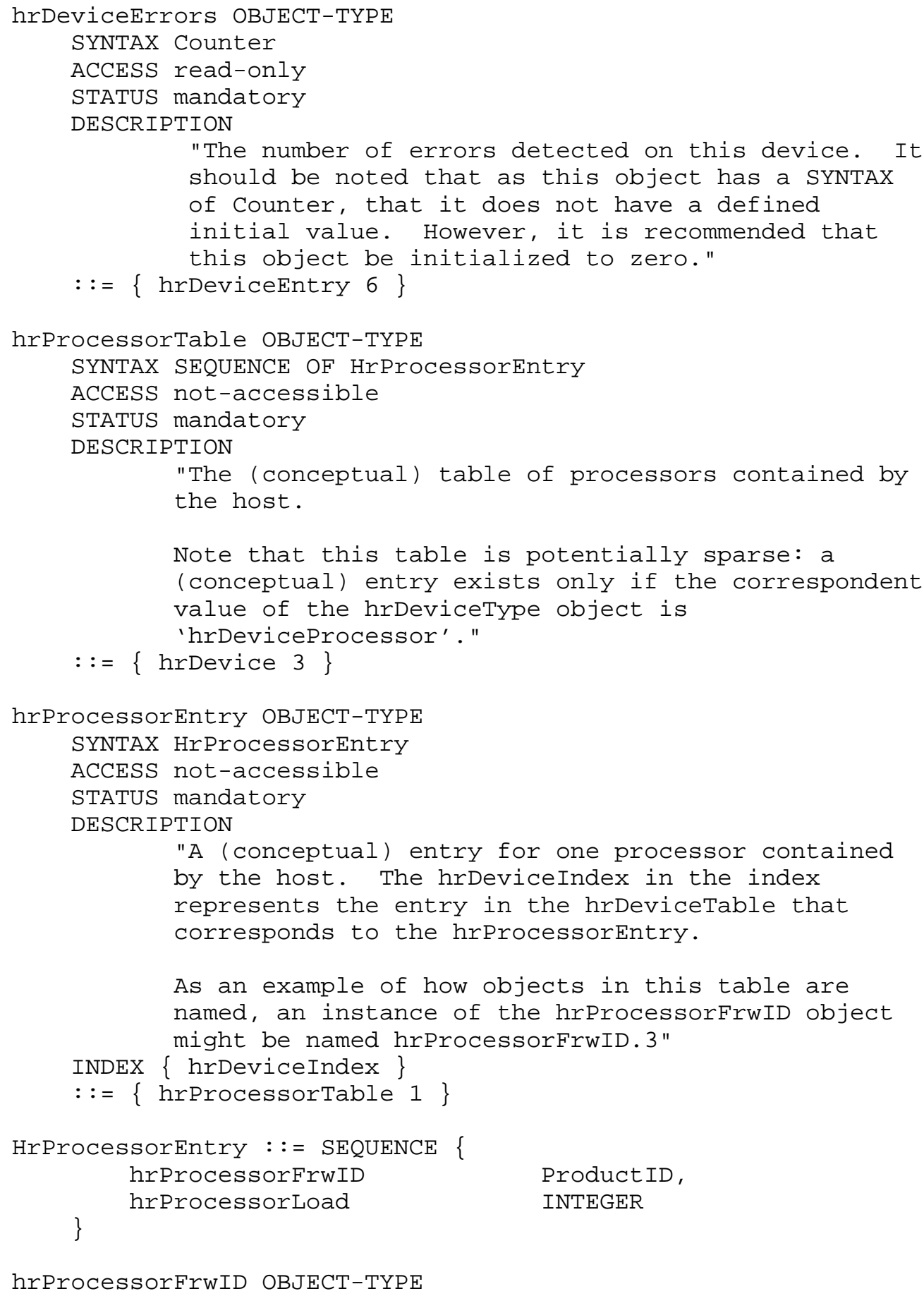




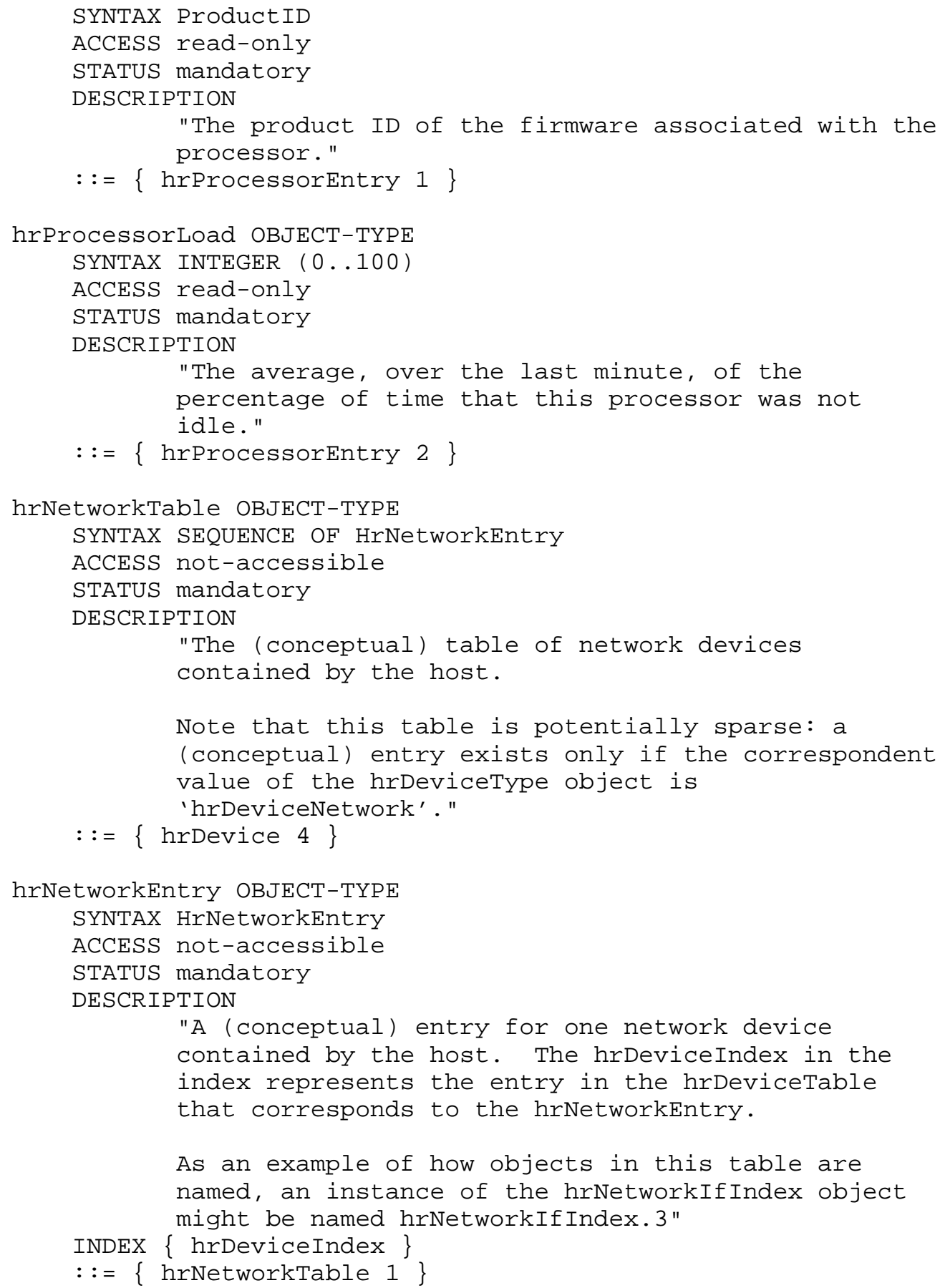




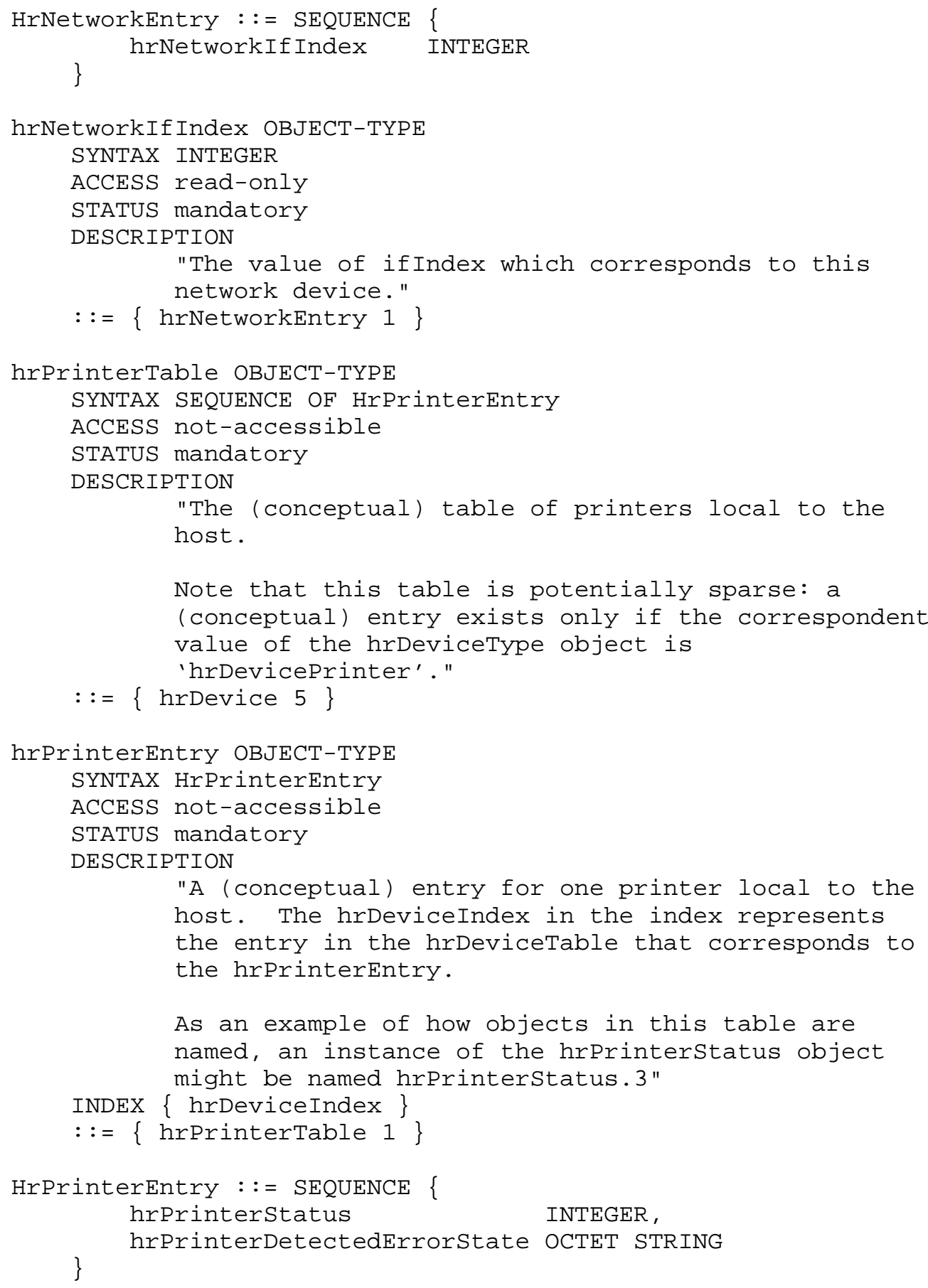




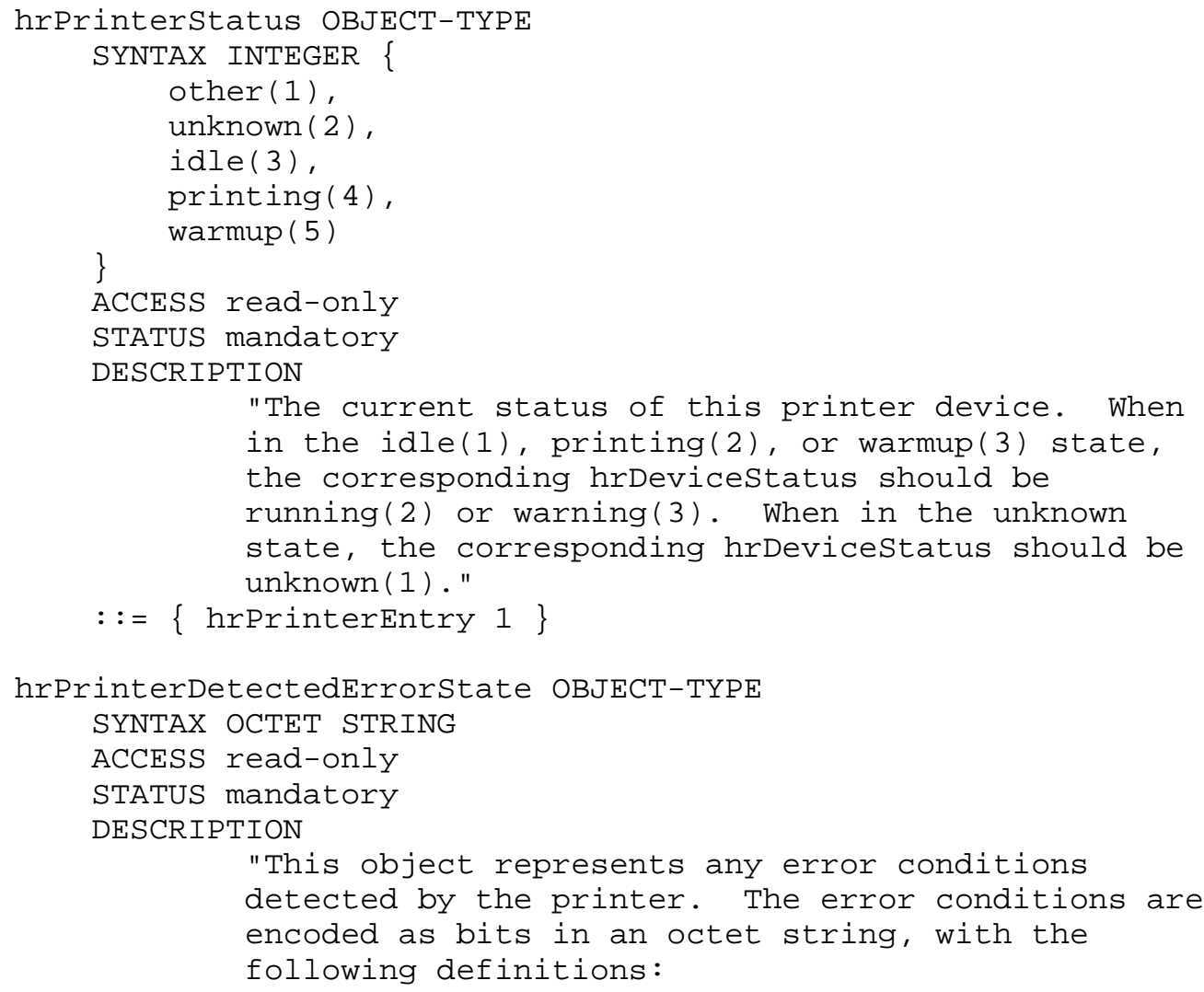




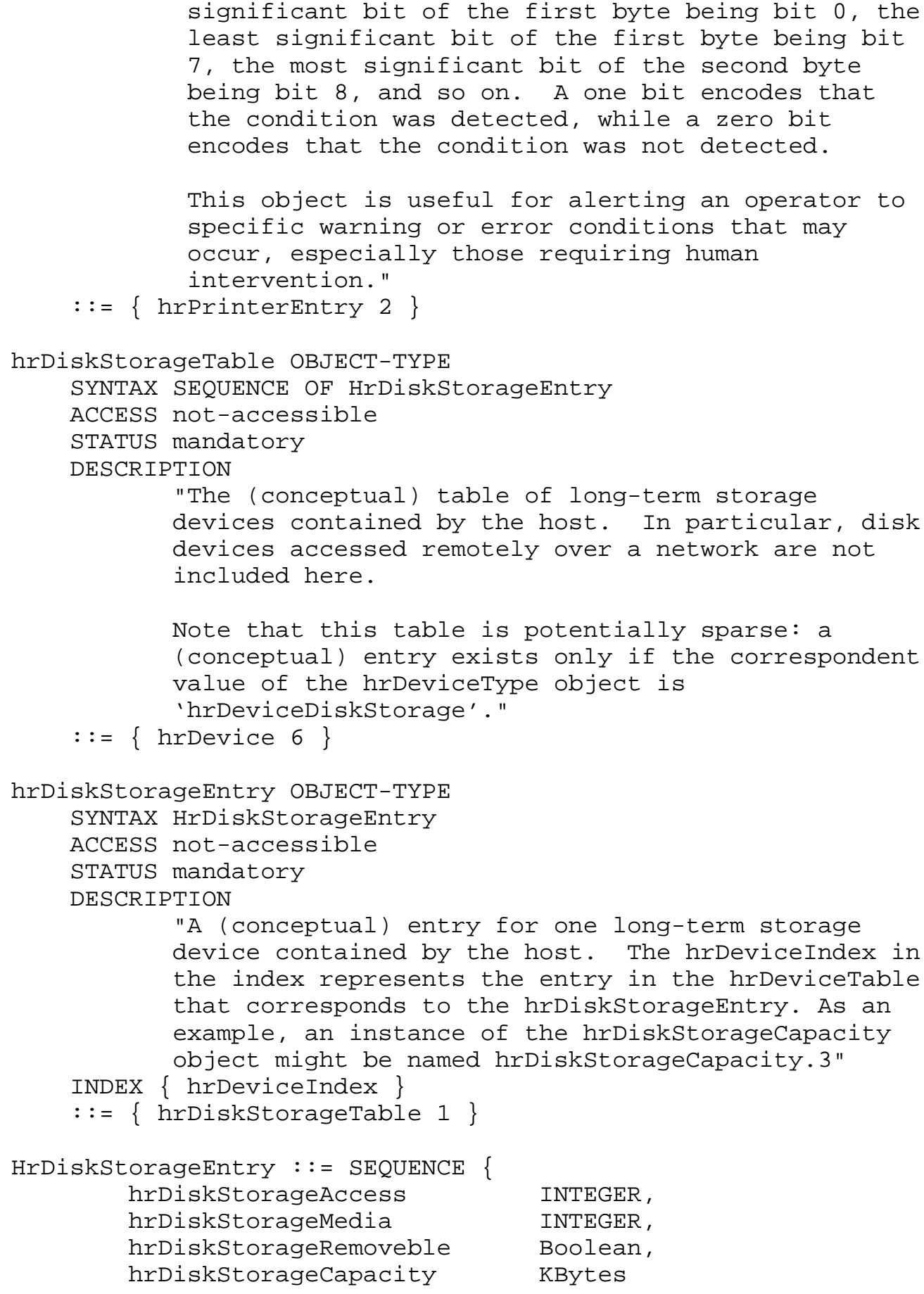


\}

hrDiskstorageAccess OBJECT-TYPE

SYNTAX INTEGER \{

readWrite (1),

\}

readonly (2)

ACCESS read-only

STATUS mandatory

DESCRIPTION

"An indication if this long-term storage device is readable and writable or only readable. This

should reflect the media type, any write-protect mechanism, and any device configuration that affects the entire device."

$::=\{$ hrDiskstorageEntry 1$\}$

hrDiskStorageMedia OBJECT-TYPE

SYNTAX INTEGER \{

other (1),

unknown (2),

hardDisk (3),

floppyDisk (4), opticalDiskROM (5), opticalDiskWORM(6), -- Write Once Read Many opticalDiskRW (7), ramDisk (8)

\}

ACCESS read-only

STATUS mandatory

DESCRIPTION

"An indication of the type of media used in this

long-term storage device."

$::=\{$ hrDiskstorageEntry 2$\}$

hrDiskStorageRemoveble OBJECT-TYPE

SYNTAX Boolean

ACCESS read-only

STATUS mandatory

DESCRIPTION

"Denotes whether or not the disk media may be

removed from the drive."

$::=\{$ hrDiskstorageEntry 3$\}$

hrDiskstorageCapacity OBJECT-TYPE

SYNTAX KBytes

ACCESS read-only

STATUS mandatory 


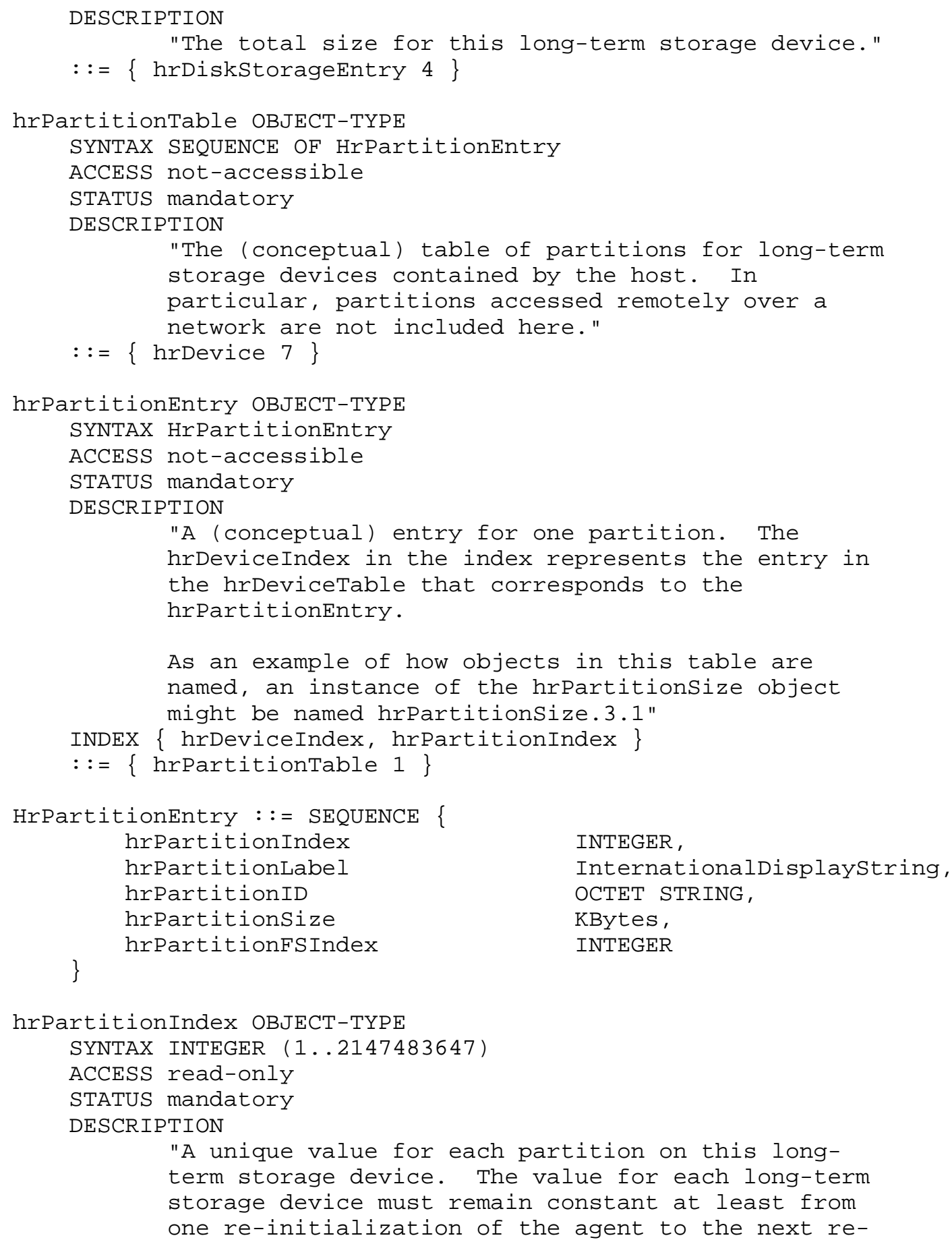




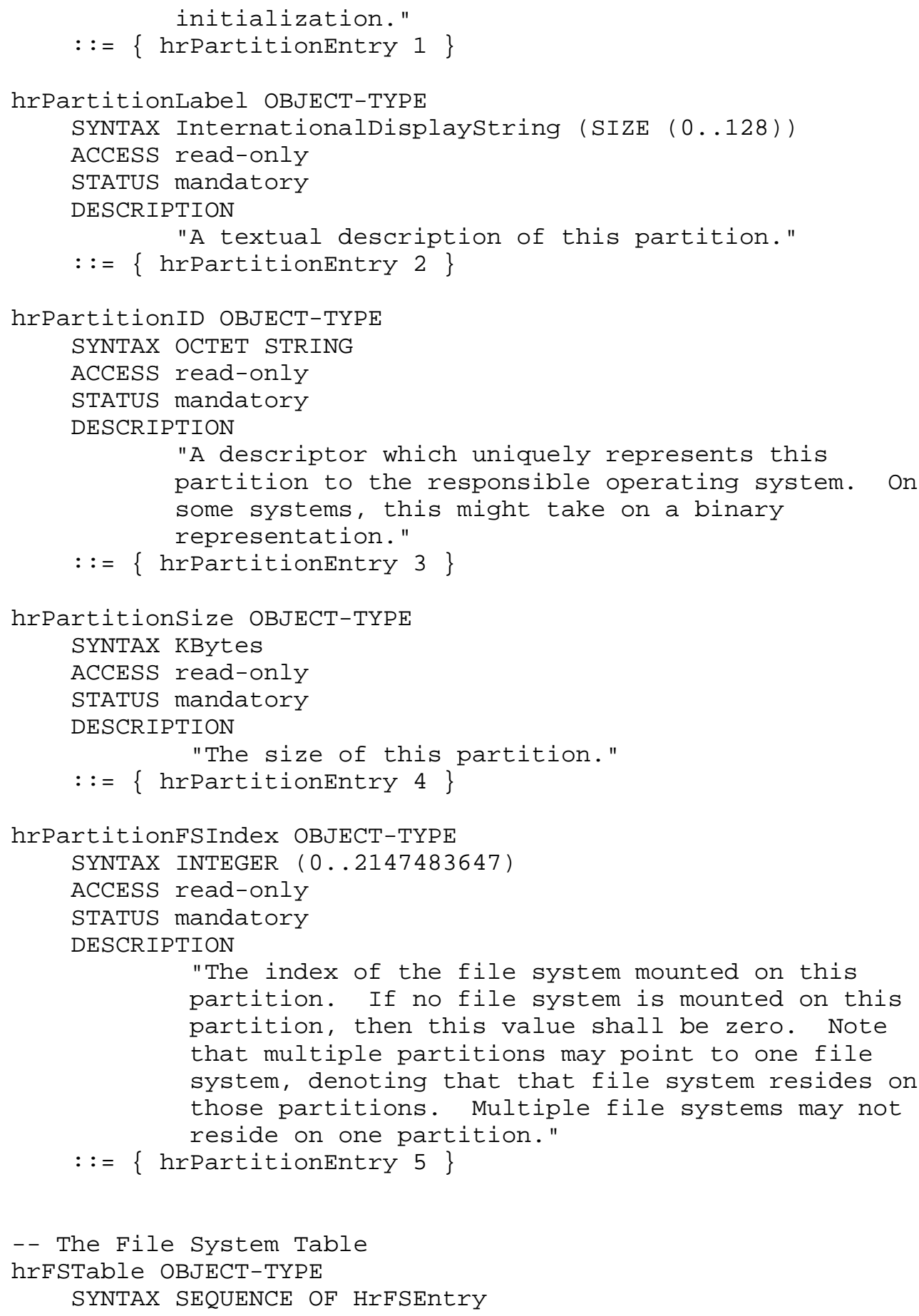




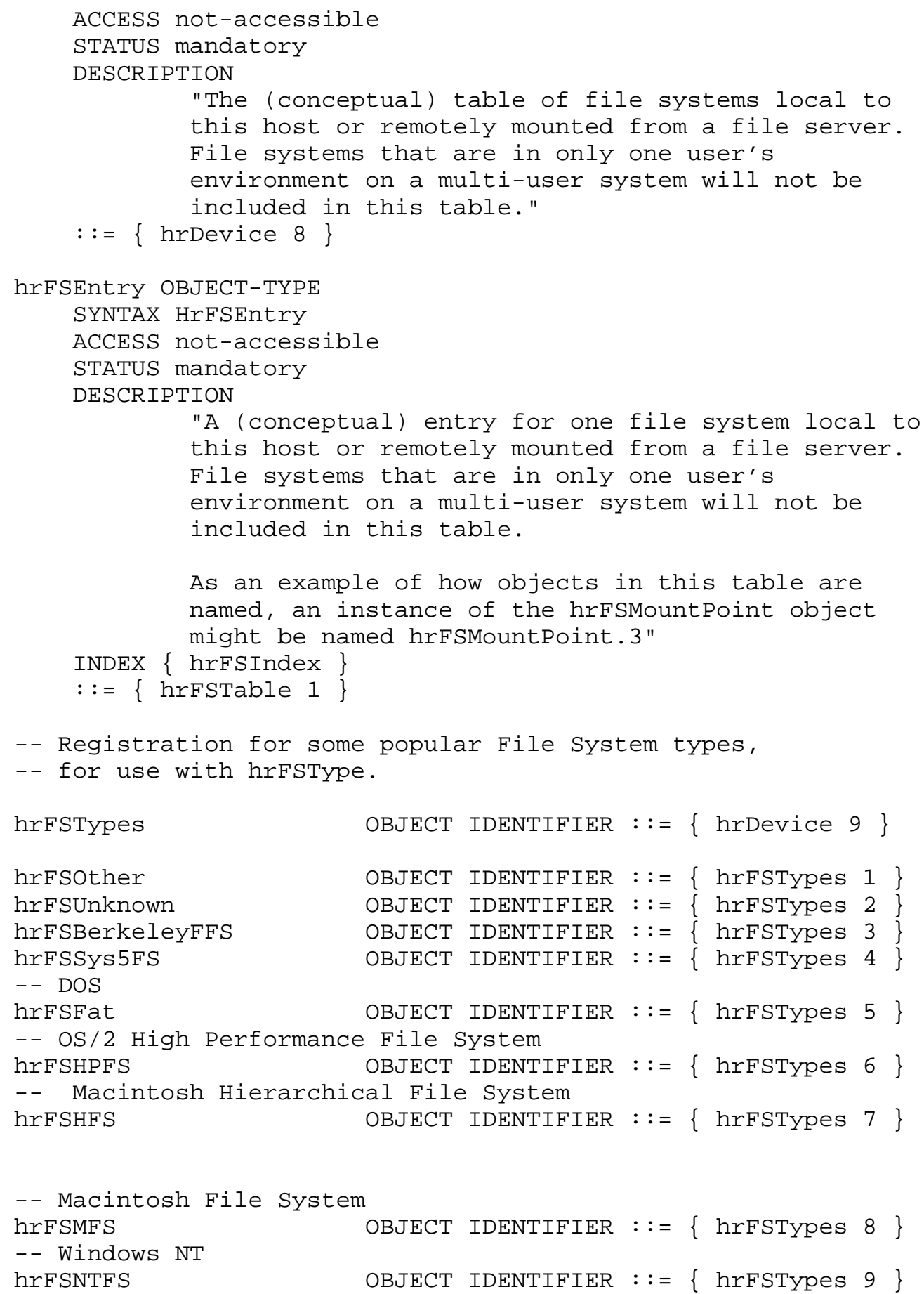




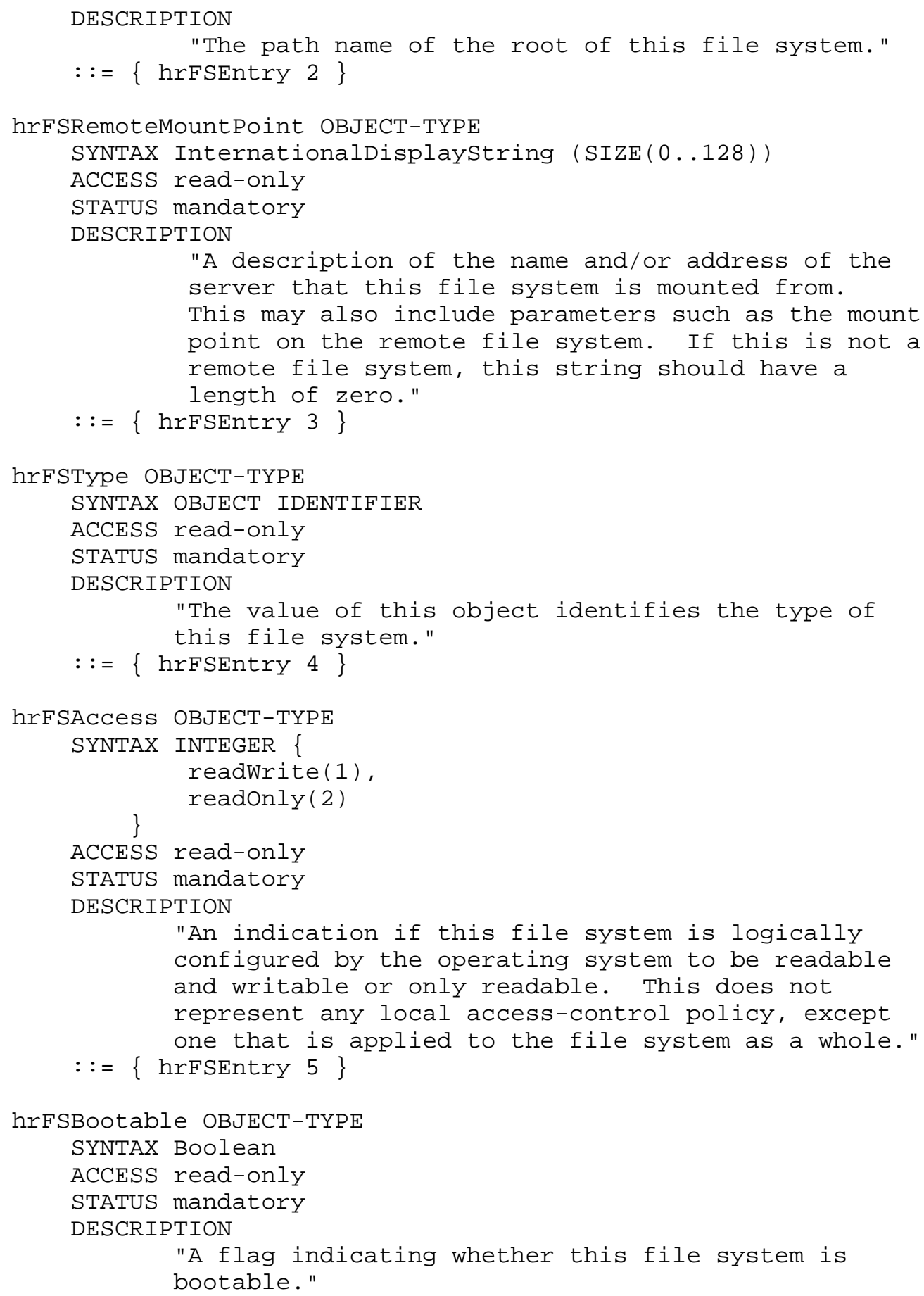

"A flag indicating whether this file system is bootable." 
$::=\{$ hrFSEntry 6$\}$

hrFSStorageIndex OBJECT-TYPE

SYNTAX INTEGER (0..2147483647)

ACCESS read-only

STATUS mandatory

DESCRIPTION

"The index of the hrStorageEntry that represents information about this file system. If there is no such information available, then this value shall be zero. The relevant storage entry will be useful in tracking the percent usage of this file system and diagnosing errors that may occur when it runs out of space."

$::=\{$ hrFSEntry 7$\}$

hrFSLastFullBackupDate OBJECT-TYPE

SYNTAX DateAndTime

ACCESS read-write

STATUS mandatory

DESCRIPTION

"The last date at which this complete file system was copied to another storage device for backup. This information is useful for ensuring that backups are being performed regularly.

If this information is not known, then this variable shall have the value corresponding to January 1, year 0000, 00:00:00.0, which is encoded as (hex)'00 00010100000000 ." $::=\{$ hrFSEntry 8$\}$

hrFSLastPartialBackupDate OBJECT-TYPE SYNTAX DateAndTime ACCESS read-write STATUS mandatory DESCRIPTION

"The last date at which a portion of this file system was copied to another storage device for backup. This information is useful for ensuring that backups are being performed regularly.

If this information is not known, then this variable shall have the value corresponding to January 1, year 0000, 00:00:00.0, which is encoded as (hex)'00 00010100000000 '."

$::=\{$ hrFSEntry 9$\}$ 


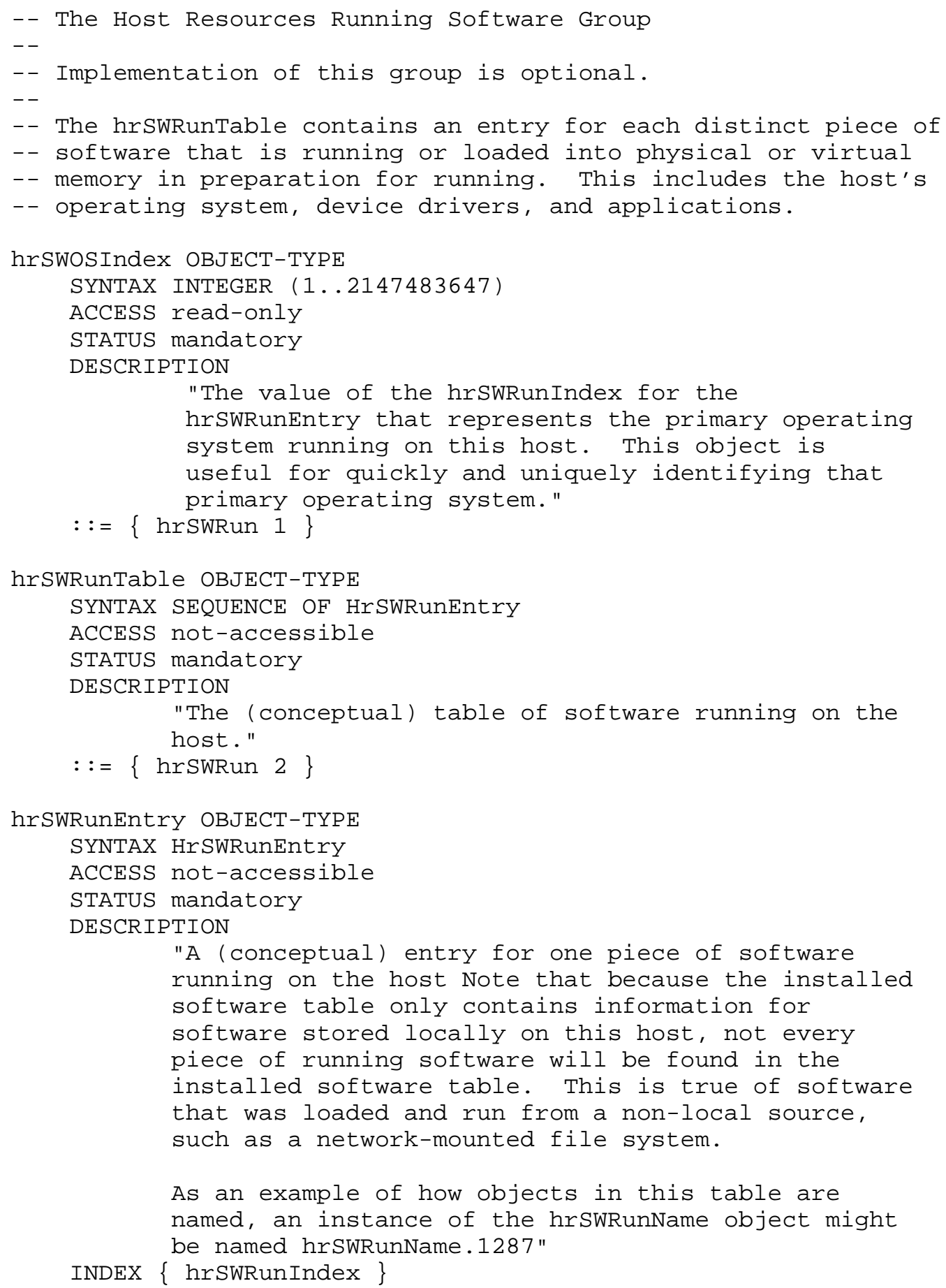




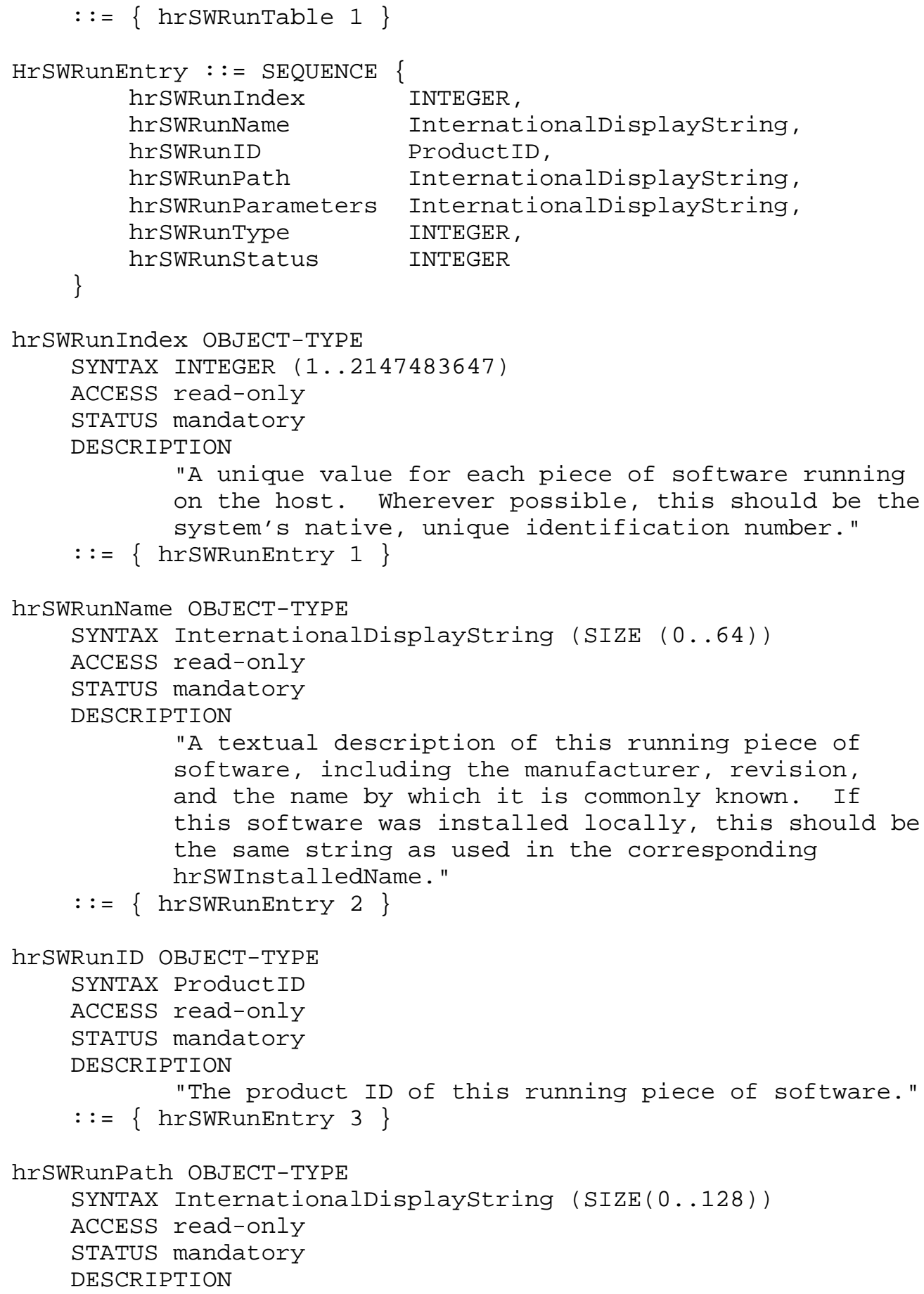




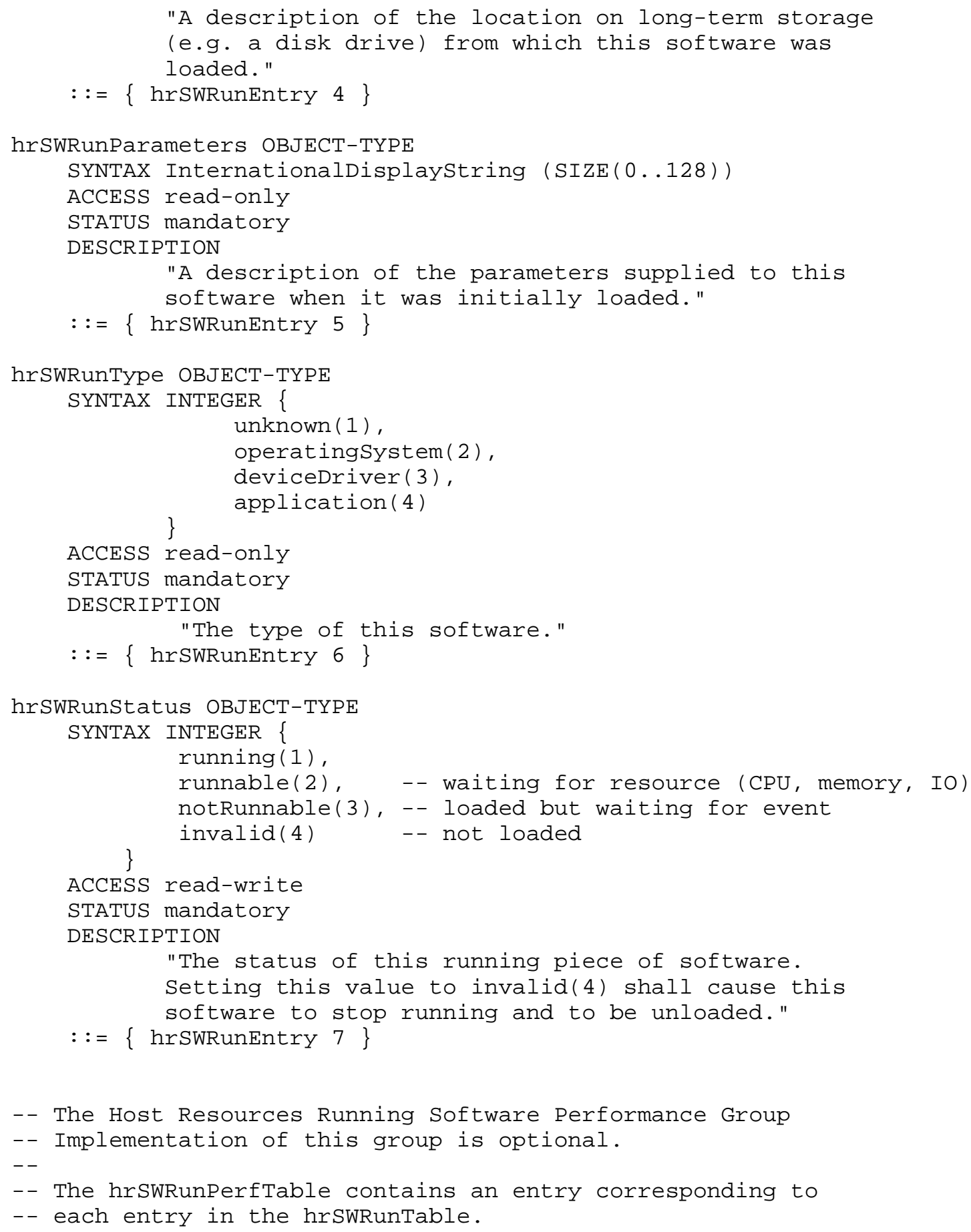




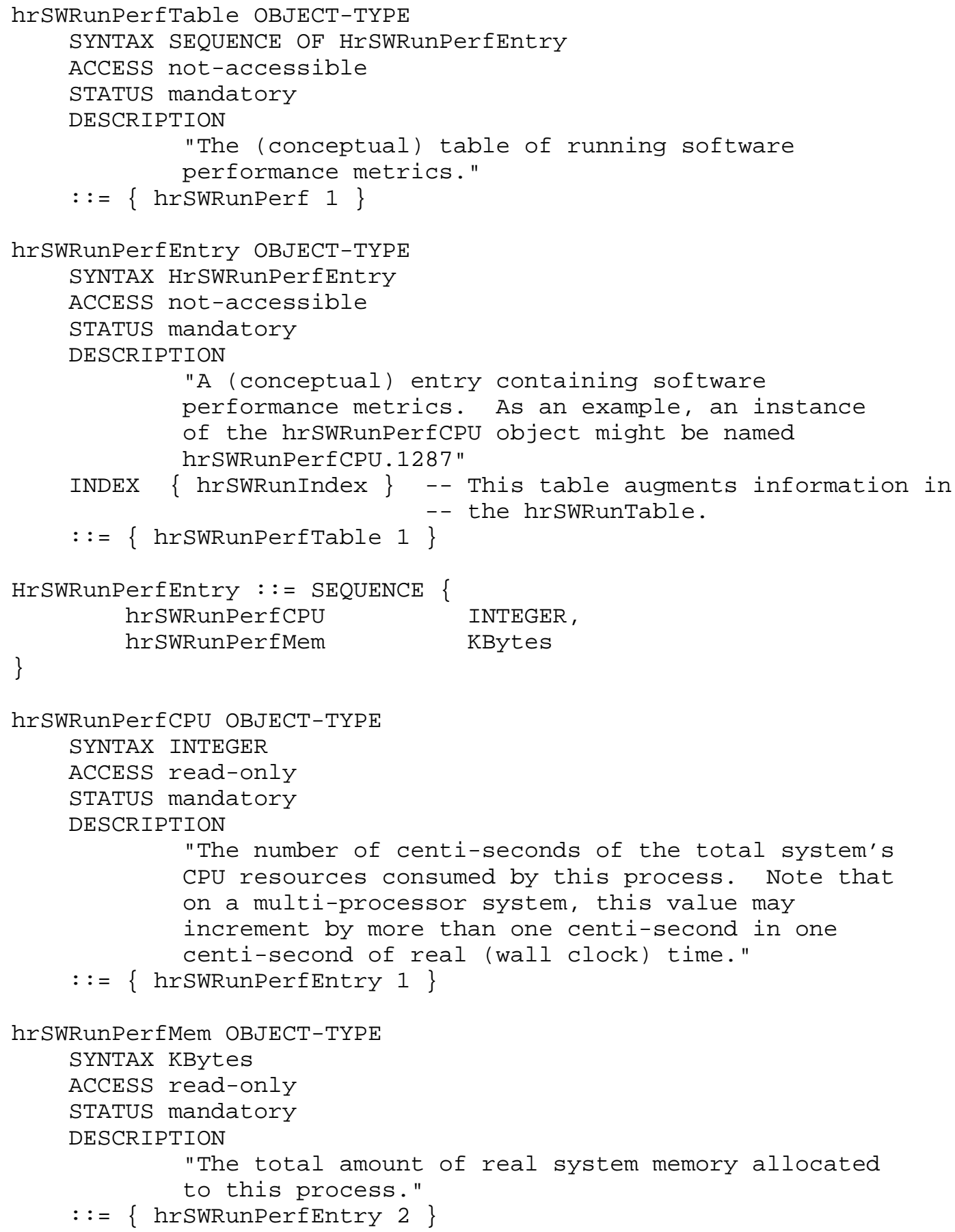




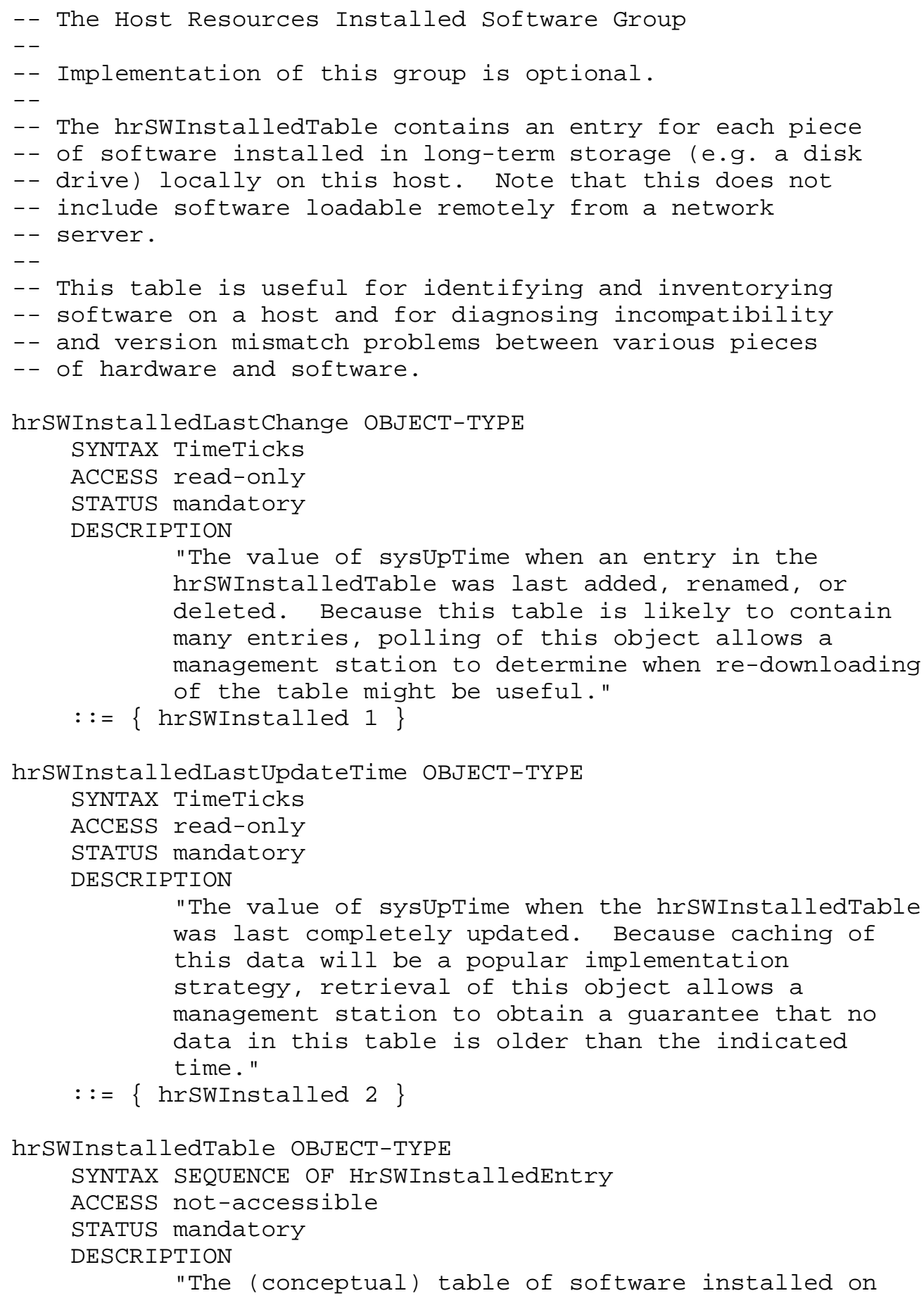




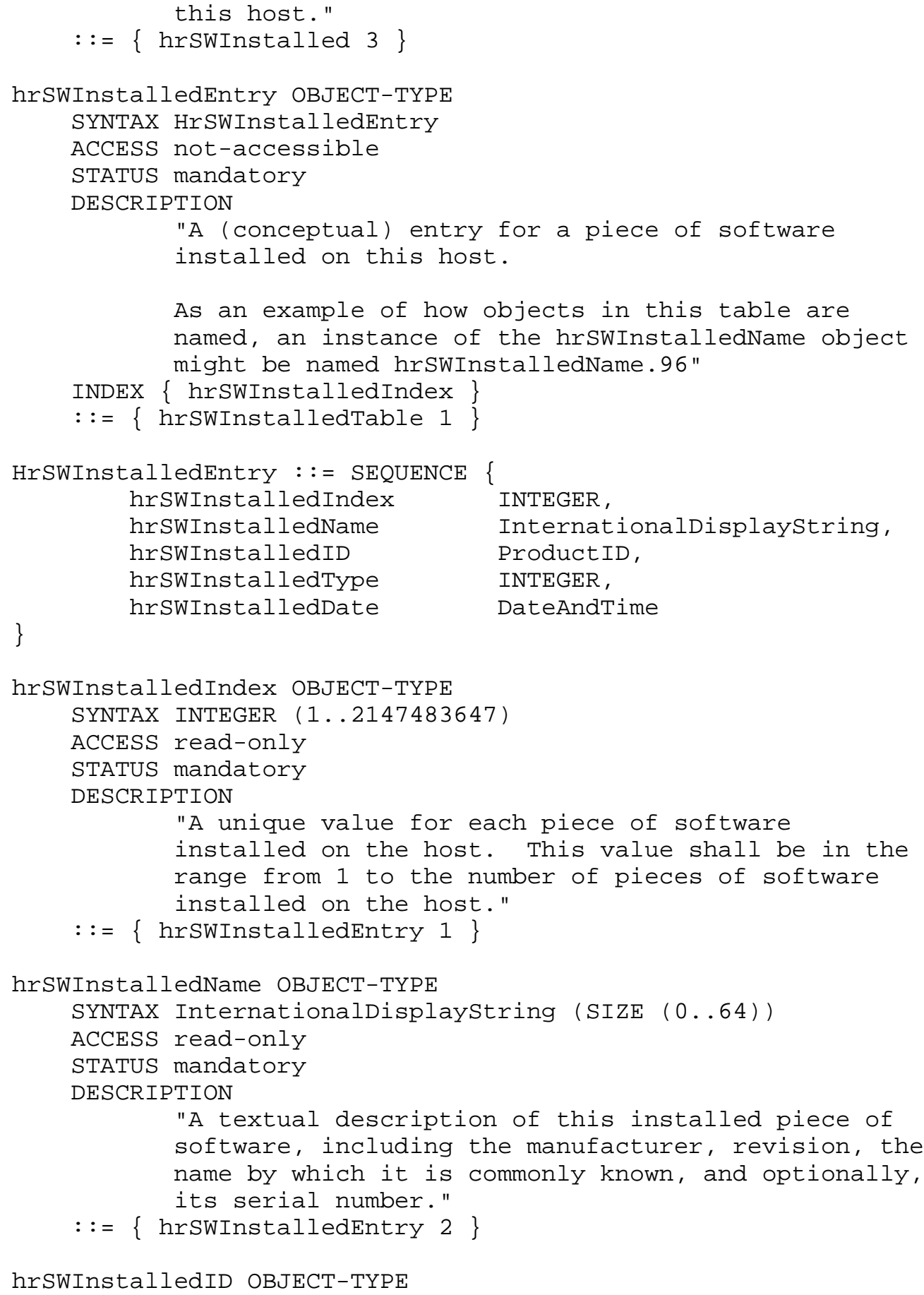




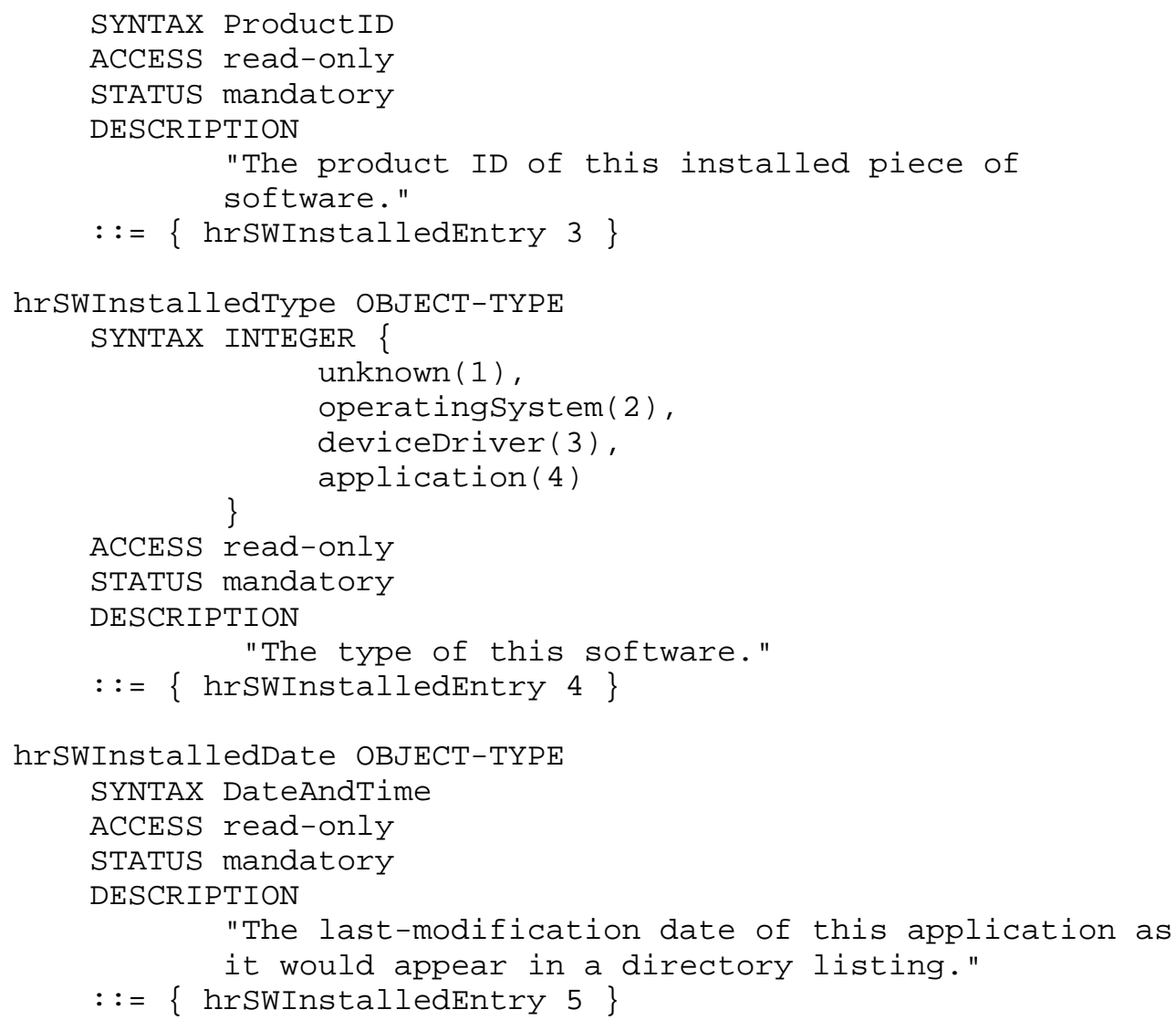

END

5. References

[1] Rose M., and K. McCloghrie, "Structure and Identification of Management Information for TCP/IP-based internets", STD 16, RFC 1155, Performance Systems International, Hughes LAN Systems, May 1990 .

[2] Rose, M., and K. MCCloghrie, Editors, "Concise MIB Definitions", STD 16, RFC 1212, Performance Systems International, Hughes LAN Systems, March 1991.

[3] McCloghrie K., and M. Rose, Editors, "Management Information Base for Network Management of TCP/IP-based internets", STD 17, RFC 1213, Performance Systems International, March 1991. 
[4] Case, J., Fedor, M., Schoffstall, M., and J. Davin, "Simple Network Management Protocol", STD 15, RFC 1157, SNMP Research, Performance Systems International, Performance Systems International, MIT Laboratory for Computer Science, May 1990.

[5] Information processing systems - Open systems Interconnection Specification of Abstract Syntax Notation One (ASN.1), International Organization for Standardization. International Standard 8824, (December, 1987).

6. Acknowledgments

This document was produced by the Host Resources MIB working group.

In addition, the authors gratefully acknowledge the comments of the following individuals:

$\begin{array}{ll}\text { Amatzia Ben-Artzi } & \text { NetManage } \\ \text { Steve Bostock } & \text { Novell } \\ \text { Stephen Bush } & \text { GE Information Systems } \\ \text { Jeff Case } & \text { SNMP Research } \\ \text { Chuck Davin } & \text { Bellcore } \\ \text { Ray Edgarton } & \text { Bell Atlantic } \\ \text { Mike Erlinger } & \text { Aerospace Corporation } \\ \text { Tim Farley } & \text { Magee Enterprises } \\ \text { Mark Kepke } & \text { Hewlett-Packard } \\ \text { Bobby Krupczak } & \text { Georgia Tech } \\ \text { Cheryl Krupczak } & \text { Georgia Tech } \\ \text { Keith McCloghrie } & \text { Hughes Lan Systems } \\ \text { Greg Minshall } & \text { Novell } \\ \text { Dave Perkins } & \text { Synoptics } \\ \text { Ed Reeder } & \text { Objective Systems Integrators } \\ \text { Mike Ritter } & \text { Apple Computer } \\ \text { Marshall Rose } & \text { Dover Beach Consulting } \\ \text { Jon Saperia } & \text { DEC } \\ \text { Rodney Thayer } & \text { Sable Technology } \\ \text { Kaj Tesink } & \text { Bellcore } \\ \text { Dean Throop } & \text { Data General } \\ \end{array}$

7. Security Considerations

Security issues are not discussed in this memo. 
8. Authors' Addresses

Pete Grillo

10915 NW Lost Park Drive

Portland OR 97229

Phone: +1 5035269766

EMail: pl0143@mail.psi.net

Steven Waldbusser

Carnegie Mellon University

4910 Forbes Ave.

Pittsburgh, PA 15213

Phone: +1 4122686628

Fax: +1 4122684987

EMail: waldbusserdcmu.edu 